In this research the most important question was: What properties must a substance have to act as a catalyzer to accelerate the hydrolysis of fats? My views on this subject I have partially expressed in my paper, "A Reagent in the Chemistry of Fats," 1 where I say that it must be a strong acid, one dissociated strongly in water giving a considerable concentration of hydrogen ions, and then it must be soluble in both fat and water and cause the one to dissolve in the other.

The only bodies that I can at present conceive to have the desired properties are sulfonic acids containing higher fatty radicals. They have the physical character of fats or oils, yet are soluble in water forming soapy solutions, and are strong acids.

As I have said, the discovery of the fatty aromatic sulfonic acids was made by accident. I had a mixture of oleic acid and benzene which I treated with an excess of sulfuric acid and then poured the mass into water. I was not surprised to find an oily layer floating on the acid water, and when I found this oil to be soluble in pure water, that also did not surprise me, as I supposed I had simply stearosulfuric acid; but when I found that after boiling for half an hour with water it still remained soluble, I felt sure that I had a sulfonic acid, and the analysis of the product confirmed my conclusion. This is the compound which has been put to practical use in the separation of glycerol and fatty acids. Instead of benzene, naphthalene is used with oleic acid in the manufacture of the commercial article. This "Saponifier," when added in the proportion of half a per cent or less to fat boiling with water in an open tank, will cause the separation of the glycerol.

Besides the compound containing the stearic radicals and a sulfonic group, the first which I found to have the catalytic property, and the fatty aromatic sulfonic acids, I have also prepared hydrolyzing reagents by treating oleic acid at $200-220^{\circ} \mathrm{C}$. with sulfur, or in the cold with sulfur chloride $\left(\mathrm{S}_{2} \mathrm{Cl}_{2}\right)$, and then oxidizing with nitric acid, potassium permanganate, bromine, or other oxidizing agent. The resulting compound contained one sulfonic and one carboxyl group and its molecular weight and other properties showed it to be a sulfonic acid of the stearic radical.

Belonging to this class of substances is a compound, cetyl sulfonic acid, described by A. Reychler two or three years ago. When I saw this description I was sure that it was also a catalyzer of the same type as my reagent, although Reychler does not mention this as one of its properties.

As this is a typical compound, it may be interesting to describe how it was prepared. partly following directions given in Reychler's paper. Cetyl alcohol, prepared by saponifying spermaceti with caustic potash and extracting the soap with petroleum ether, was converted into the iodide by dropping iodine into a heated mixture of the alcohol and red phosphorus. This iodide, after purification, was converted into the sulfhydrate by treating with alcoholic potassium sulfhydrate and the cetyl sulfhydrate converted into cetyl sulfonic acid by oxidizing with potassium permanganate, the excess of which was reduced with sodium sulfite. The cetyl sulfonates could be freed from most of the foreign matter by taking up with hot water from which sodium cetyl sulfonate crystallized on cooling. This was dried, extracted with petroleum ether, then dissolved in water and treated with hydrochloric acid. The salted out cetyl sulfonic acid was dissolved in ether and remained in a nearly pure state as a residue on evaporating the ether. It can be further purified by crystallizing its sodium salt from dilute alcohol and there can be no doubt of its composition. It is a simple sulfonic acid of a hydrocarbon of the paraffine series.

Reychler explains some peculiar properties of this compound in this way: the sulfonic radical tends to make it very soluble in water, while the long hydrocarbon chain has just the reverse effect. The result is that it forms colloidal solutions. Fahrion,

$$
\text { 1 J. Am. Chem. Soc., } 28 \text { (1906), } 196 .
$$

in reviewing Reychler's article, calls the cetyl sulfonic acid, "Reychler's hydrogen soap" and that term, hydrogen soap, is as good a definition as I can think of to cover the whole group of compounds which catalytically induce hydrolysis of fats on the principle of my discovery. An alkaline soap plus hydrogen ions would no doubt have the same effect if it were possible to have such a combination.

Besides its use in hydrolyzing fats, my catalytic agent has been applied to other less important ones. A catalyst, which would accelerate the hydrolysis of an ester in the presence of an excess of water, would also accelerate the esterification of a fatty acid and an alcohol on the removal of the water formed. Fatty acids can be made to combine with glycerol and other alcohols of high boiling point by simply treating a mixture of the two with a small quantity of the reagent and evaporating the water formed at $100^{\circ} \mathrm{C}$.

The solubility of sulfo-fatty acids in both fat and water leads to a method of separating solid and liquid fatty acids of which I have made some application. If a smail quantity of the sulfonic reagent is dissolved in melted mixed fatty acids and the mixture allowed to cool, the solid acids will crystallize out pure and the liquid acids will contain the sulfo-fatty acids, being thus rendered slightly soluble in water, and on treating with water, can be washed out from the mixture, partly in solution but mainly as an emulsion.

Patents have recently been obtained by Grigory Petroff of Russia on a reagent obtained as a by-product in refining petroleum with fuming sulfuric acid. This is a sulfonic acid of hydrocarbon radicals, probably not of the paraffine series. It is a very efficient catalyzer in the hydrolysis of fats, for which purpose it is now largely used.

Wyоmng, Oнго

\section{THE TWITCHELL PROCESS AND THE GLYCERINE TRADE}

By A. C. LANGMUIR

Some years ago a manufacturer asked my assistance in developing a chemical process. He wanted a process which would "cost nothing to work, would take nothing out of the product and would put nothing in." This man had heard nothing of catalytic methods, but his conception of the ideal chemical process which he was requiring corresponded closely to the modern catalytic methods such as the platinum contact process for the manufacturer of sulfuric acid, which costs nothing to operate, once the contact mass is prepared, and which brings about the combination of sulfur dioxide and oxygen without adding an impurity which must be subsequently taken out and without removing something, which would lower the yield.

The Twitchell catalytic process goes far toward meeting this ideal in the saponification or breaking up of fats and oils into their constituents-glycerine and fatty acids. Twitchell's in. vention, U. S. Patent 601,603 , July 17,1897 , was made at a time when catalysis was not the universal subject of attention it is to-day. The Badische process for the catalytic production of sulfuric acid had not been published and catalysis was a new thing in chemical industry.

Twitchell's work has been characterized by a sound appreciation of the work of physical and organic chemists and his analytical and technical methods have been worked out, not empirically, but from theoretical premises. Thus the Twitchell method for the determination of rosin in mixtures with fatty acids as in soap analysis, rests upon the principle of the esterification of the fatty acids when treated with hydrochloric acid gas in presence of absolute alcohol and his discovery that resin acids when so treated refused to combine with the alcohol and could subsequently be separated as pure rosin. Although published in $189 \mathrm{I}$, this method stands to-day as the most accurate process for the determination of rosin in presence of fatty acids.

Again in 1897 we find him bringing out a method for the: 
separation of saturated and unsaturated fatty acids based upon the solubility of fatty acids with double bonds, such as oleic acid, in concentrated sulfuric acid at ordinary temperatures with the formation of sulfo-fatty acids soluble in water; the saturated fatty acids such as stearic acid are unacted on. In I9r4 Twitchell makes use of the principle of the equal depression of the freezing point for equal molecular proportions and determines the composition of mixtures of fatty acids by the observation of the freezing point in the Beckmann apparatus. To test whether a given sample of fatty acid is identical with a fatty acid of known character he adopts the novel plan of adding a portion of the known acid and noting whether there is any change in the melting point of the mixture.

Possibly his investigation of the action of concentrated sulfuric acid on fatty acids led to his discovery of the catalytic action of these sulfo-fatty acids in bringing about the decomposition of fats by water, as little as 0.5 per cent splitting up the fats almost completely on digestion with water.

The prevailing method for the manufacture of soap consists in the saponification of the fat by boiling with caustic soda lye. The fatty acids combine with the soda as soap, which is separated by the addition of salt and comes to the surface in the molten condition. The glycerine is set free and remains dissolved in the spent lye. It is contaminated by the presence of the salt used to render the soap insoluble. During the concentration of the spent lye for the recovery of the glycerine a large quantity of salt is thrown out, making the evaporation somewhat difficult, although this difficulty has been largely eliminated of late years by the use of properly designed evaporators working in vacuo. The final crude glycerine is a saturated solution of sodium chloride and some sulfate together with sodium salts of the lower fatty acids in glycerine and water. The crude averages about 80 per cent glycerine and contains about ro per cent mineral salts.

The glycerine refiner distills this crude soap lye glycerine with superheated steam in vacuo for the production of dynamite glycerine and chemically pure glycerine. The presence of so large a quantity of salt raises the boiling point of the glycerine and reduces its vapor tension so that the output is reduced. Furthermore, the salt accumulates in the still, producing finally a semi-solid mass of salt and glycerine together with tarry matter which constitutes the "foots" of the glycerine trade. It is a difficult matter to extract all the glycerine from this residue without undue loss. The treatment of the "foots" is one of the problems of the glycerine trade.

The glycerine distiller therefore prefers a crude glycerine which is free from this large admixture of salt. Up to the early nineties all the crude glycerine refined consisted of what is termed "saponification" or "candle crude." It was the byproduct of the candle factory and was produced by the breaking up of fat in autoclaves by heating under pressures of $200 \mathrm{lbs}$. and more with water and a little lime. The fat was split into fatty acids and glycerine directly and the latter was a product of considerable purity, containing about 88 per cent glycerine and less than r.o per cent of mineral matter. With the greatly increased demand for glycerine for explosives, the soap makers, who formerly discarded their spent lyes, found it profitable to work them up and to-day scarcely a soap plant can be found that does not recover its glycerine as a by-product. This change in the business compelled refiners to develop methods for distilling soap lye crude glycerine and eventually the larger refiners were working mainly on soap lye rather than candle crudes although there were still a number of glycerine refiners who worked exclusively on candle crudes. Saponification crudes free from salt have always been in greater demand and have commanded a higher price per unit of glycerine. For several years before the advent of the Twitchell process it looked as if saponification glycerines would practically disappear as a raw material in glycerine refining, but with the success of this process, the Twitchell saponification crudes made their appearance and are now a factor of very considerable importance.

The ideal of the glycerine refiner has thus been the production of a saponification rather than a soap lye crude by the soap manufacturer. It has also been the hope of the soap trade to obtain directly by the deglycerinizing of the fat a relatively pure glycerine as a by-product and free fatty acids which could be combined with the cheap alkali, carbonate of soda, to make soap instead of the relatively expensive caustic soda. To meet this need have been developed the Twitchell process, the Krebitz lime saponification method and the Connstein ferment process.

The Twitchell process is characterized by its simplicity, and the low cost of the plant. Prior to Twitchell's discovery free fatty acids could be obtained only by the autoclave process, involving a heavy capital outlay for copper apparatus, which could be used only in small units and at a high temperature and pressure, making the operation expensive and somewhat dangerous. The autoclave saponification was used only in the preparation of fatty acids for candle manufacture as the process was too costly to be used in the production of fatty acids to be used in soap. The Twitchell process is effected in loosely closed wooden tanks by digestion with water and as little as 0.5 per cent of the reagent and at a temperature not exceeding that of exhaust steam. The reaction may be carried out on a scale limited only by the size of the tank. The Twitchell process has made possible the saponification of fats on a huge scale for the direct production of free fatty acids and saponification glycerine. When combined with the distillation of the fatty acids for the improvement of their purity and color it has opened up for the soap trade the use of low-grade fats such as garbage grease and cottonseed oil foots, the distilled fatty acids being combined directly with carbonate of soda to produce light colored soaps, and the glycerine being made available for the manufacture of dynamite glycerine. In this way the Twitchell process has benefited the glycerine trade in these days of glycerine scarcity by opening up new sources of supply which were previously unavailable.

In Germany, Austria, Belgium, Holland and Scandinavia the larger soap plants prepare soap from fatty acids directly and it is stated that in most of them the Twitchell process is used for the saponification of the fats.

The crude glycerine prepared by the Twitchell process comes to us from all parts of the world in normal times. There is no evidence that it is any less pure than the saponification glycerines prepared by other processes provided the fat used as a raw material is reasonably pure. Of course a crude glycerine manufactured from a low-grade fat will fall behind a crude made from good tallow, but this is the fault of the fat and not of the Twitchell process.

\section{VAN BrUnt Street \\ BROOKLYN, NEW YORE \\ THE TWITCHELL PROCESS IN THE SOAP AND CANDLE INDUSTRY}

By MARTIN H. ITTNER

It is essential in the candle industry to have a satisfactory method for separating the fatty acids, as such, from fats and oils. In the soap industry such a method, although it may be considered highly desirable, is not absolutely essential since very satisfactory methods of direct saponification by alkali with the formation of soaps have long been utilized.

In recent years there has been an ever increasing demand for fats and oils and for glycerine, resulting in greatly increased market prices for these articles. The market for soap and candle materials is directly related to the market for fats and oils for edible purposes, and an increased demand from either source usually results in higher prices for all fatty materials. 